\title{
Atividade antimicrobiana de óleos essenciais em bactérias patogênicas de origem alimentar
}

\author{
VALERIANO, C. ${ }^{1 *}$; PICCOLI,R.H. ${ }^{2}$; CARDOSO,M.G. ${ }^{3}$; ALVES,E. ${ }^{4}$ \\ ${ }^{1}$ Universidade Federal de Lavras, Departamento de Biologia, ${ }^{2}$ Departamento de Ciência dos Alimentos, \\ *carol_valeriano@yahoo.com.br ${ }^{3}$ Departamento de Química, ${ }^{4}$ Departamento de Fitopatologia, Campus Universitário, \\ Caixa Postal 3037, CEP: 37200-000, Lavras-Brasil *carol_valeriano@yahoo.com.br
}

RESUMO: Objetivou-se identificar e quantificar os constituintes e avaliar a atividade antimicrobiana dos óleos essenciais de Mentha piperita, Cymbopogon citratus, Ocimum basilicum e Origanum majorana contra cepas de Escherichia coli enteropatogênica, Salmonella enterica Enteritidis, Listeria monocytogenes e Enterobacter sakazaki. A obtenção dos óleos essenciais foi realizada a partir de folhas secas, empregando-se a técnica de hidrodestilação e utilizando-se a aparelho de Clevenger modificado. A atividade antibacteriana dos óleos essenciais foi determinada pelo método de difusão em ágar. Observou-se que os óleos essenciais inibiram o crescimento bacteriano, mas a efetividade foi variada. Entre os óleos essenciais testados, $M$. piperita apresentou maior atividade antibacteriana para E. coli, (8.106 UA $\left.\mathrm{mL}^{-1}\right)$ quando comparada as demais bactérias, atividade moderada para Salmonella enterica Enteritidis e Enterobacter sakazakii (1.706 e 3.200 UA mL $\mathrm{m}^{-1}$ respectivamente) e baixa atividade para Listeria monocytogenes $\left(106,67 \mathrm{UA} \mathrm{mL}{ }^{-1}\right)$. Já óleo essencial de Cymbopogon citratus apresentou maior atividade antimicrobiana frente a $E$. coli (9.386 UA mL-1) e atividade moderada frente a Enterobacter sakazakii, Salmonella enterica Enteritidis e Listeria monocytogenes (2.773 UA mL-1 para ambas). Ocimum basilicum apresentou maior atividade antibacteriana frente E. coli e Enterobacter sakazakii (6.826 e $8.106 \mathrm{UA} \mathrm{mL}^{-1}$ respectivamente), moderada atividade frente a Salmonella enterica Enteritidis (1.600 UA mL-1) e não apresentou atividade frente a Listeria monocytogenes. Origanum majorana também foi testado neste estudo e apresentou maior atividade antimicrobiana frente $E$. coli $\left(5.973 \mathrm{UA} \mathrm{mL}^{-1}\right)$, atividade moderada para Salmonella enterica Enteritidis e Enterobacter sakazakii (1.706 e $2.346 \mathrm{UA} \mathrm{mL}^{-1}$, respectivamente) e não apresentou atividade para Listeria monocytogenes .

Palavras-chave: monoterpenos, patógenos de origem alimentar, propriedades antibacterianas

ABSTRACT: Antimicrobial activity of essential oils against sessile and planktonic pathogens of food source. The objective of this work was to identify and quantify the constituents, and to evaluate the antimicrobial activity of the essential oils from Mentha piperita, Cymbopogon citratus, Ocimum basilicum and Origanum majorana, against enteropathogenic Escherichia coli, Salmonella enterica Enteritidis, Listeria monocytogenes and Enterobacter sakazakii. The essential oils were obtained from dried leaves by using the hydrodistillation technique and the modified Clevenger apparatus, and their bacterial activity was determined by using the agar diffusion technique. The essential oils inhibited bacterial growth, but their effectiveness was varied. Among the essential oils tested, that from M. piperita showed a greater antimicrobial activity against $E$. coli (8.106 UA $\left.\mathrm{mL}^{-1}\right)$, moderate activity for $S$. enterica Enteritidis and E. sakazakii (1.706 e $3.200 \mathrm{UA} \mathrm{mL}^{-1}$ respectively) and low activity for $L$. monocytogenes $\left(106,67 \cup A \mathrm{~mL}^{-1}\right)$. However, the essential oil from $C$. citratus presented a greater antimicrobial activity against $E$. coli $\left(9.386 \mathrm{UA} \mathrm{mL}^{-1}\right)$ and a moderate activity against E. sakazakii, S. enterica Enteritidis and L. monocytogenes (2.773 UA $\mathrm{mL}^{-1}$ for both). The essential oil from $O$. basilicum showed a greater antimicrobial activity against E. coli and E. sakazakii ( 6.826 e $8.106 \mathrm{UA} \mathrm{mL}^{-1}$ respectively), moderate activity against $S$. enterica Enteritidis (1.600 UA $\left.\mathrm{mL}^{-1}\right)$, and was inactive against $L$, monocytogenes. Origanum majorana, which was also tested in our work, showed a greater antibacterial activity against $E$. coli, (5.973 $\mathrm{UA} \mathrm{mL}{ }^{-1}$ ) moderate activity against $S$. enterica Enteritidis and $E$. sakazakii (1.706 e $2.346 \mathrm{UA} \mathrm{mL}^{-1}$, respectively), and was inactive against $L$. monocytogenes.

Key words: monoterpenes, pathogenic food source, antibacterial properties

Recebido para publicação em 25/03/2010

Aceito para publicação em 25/02/2011

Rev. Bras. PI. Med., Botucatu, v.14, n.1, p.57-67, 2012. 


\section{INTRODUCÃO}

Patógenos de origem alimentar são amplamente diversos na natureza e continuam sendo a maior causa de problemas mundiais de saúde pública em países desenvolvidos e em desenvolvimento. Esses agentes são responsáveis por considerável morbidade e mortalidade, além de resultar em custos com cuidados médicos, perda de produtividade e controle pela indústria de alimentos (Frantamico et al., 2007; Nedorostova et al., 2009). Entre os mais sérios patógenos de origem alimentar estão Salmonella sp., Listeria monocytogenes e E. coli, os quais respondem pelo maior número de casos de doenças e mortes (Ricke et al., 2005; Oussalah et al., 2007).

Para reduzir doenças e danos econômicos causados por microrganismos patogênicos, o uso de produtos naturais como compostos antimicrobianos parece ser uma maneira interessante de controlar a presença de bactérias patogênicas e estender a vida de prateleira de alimentos processados (Cowan, 1999; Filoche et al., 2005; Nedorostova et al., 2009), principalmente devido à prevalência de microrganismos resistentes a antissépticos e antibióticos convencionais e também pelo aumento do conceito popular sobre qualidade de alimentos e do potencial impacto negativo dos aditivos sintéticos na saúde. Assim, a crescente demanda do consumidor por produtos naturais efetivos e seguros tem levado a investigações com relação aos efeitos de fitoquímicos. Entre esses estudos é crescente a pesquisa com relação aos óleos essenciais (Dorman \& Deans, 2000; Draughon, 2004; Filoche et al., 2005).

Os óleos essenciais e os componentes são considerados os agentes antimicrobianos mais importantes presentes em plantas; eles podem também apresentar atividade antioxidante e antiinflamatória (Cowan, 1999). Os óleos essenciais originam-se do metabolismo secundário das plantas, sendo constituídos por uma mistura de compostos, principalmente monoterpenos, sesquiterpenos, e derivados oxigenados (álcoois, aldeídos, ester, éteres, cetonas, fenóis e óxidos). Outros compostos voláteis incluem fenilpropanoides e substâncias contendo enxofre ou nitrogênio (Bajpai et al., 2008).

Estudos da atividade antibacteriana de óleos essenciais frente à patógenos de origem alimentar tem sido realizado, principalmente, in vitro (Oussalah et al., 2007) e, recentemente pela aplicação simultânea da bactéria e de óleos essenciais em produtos alimentícios (Solomakosa et al., 2008; Oliveira et al., 2011). Os óleos essenciais derivados de Mentha piperita, Cymbopogon citrarus, Origanum majorana, Ocimum basilicum tem sido estudados para este propósito.

A atividade antibacteriana do óleo essência de Cymbopogon citratus tem sido relatada contra frente a vários patógenos (Cimanga, et al., 2002). O óleo essencial é basicamente constituído de citral (70 a $85 \%$ v/v) (Ferreira \& Fonteles, 1989), mistura isomérica de neral (citral $B$ ou isômero $Z$ ) e geranial (citral A ou isômero E) (EIFattah et al., 1992). Além destes, pode conter mirceno e outros compostos minoritário como, por exemplo, geraniol, cimbopogol, limoneno e dipenteno (Martins et. al., 2003). Óleo essencial de Mentha piperita tem sido também relatado por possuir atividade, antibacteriana, antiviral e antifúngica, sendo esta atividade associada principalmente aos compostos majoritários mentol, mentona, acetato de metila, iso-mentona (Singh, et al., 2011). O óleo essencial de O. majorana é rico em compostos bioativos como terpinen-4-ol, sabineno, acetato de linalol, $\gamma$-terpineno e linalol. Estes compostos exibem atividade antibacteriana elevada e amplo expectro de ação (Sellami, et al., 2009). Em adição o gênero Ocimum basilicum é conhecido por possuir uma gama de atividades biológicas, tais como repelente de insetos, inibidora de nematóides, antibacteriana, antifúngica e atividades antioxidantes (Lee et al., 2005). Compostos como, linalol, metil chavicol, cinamato de metila, metil eugenol e mistura destes são comumente os principais componentes dos óleos essencial das espécies e variedades de Ocimum (Telci et al., 2006).

O presente estudo foi realizado com o objetivo de identificar e quantificar os constituintes e avaliar a atividade antibacteriana de óleos essenciais de hortelã-pimenta (Mentha piperita), capim-limão (Cymbopogon citratus), manjerona (Origanum majorana), e manjericão (Ocimum basilicum) frente os patógenos de origem alimentar Escherichia coli enteropatogênica CDC 0126, Salmonella entérica Enteritidis S 64, Listeria monocytogenes ATCC 19117 e Enterobacter sakazakii ATCC 29004.

\section{MATERIALE MÉTODO}

\section{Material vegetal}

Os materiais vegetais utilizados para a extração de óleo essencial foram folhas de hortelãpimenta (Mentha piperita L.) e de capim-limão (Cymbopogon citratus (D.C.) Stapf), manjerona (Origanum majorana L.) e manjericão (Ocimum basilicum L.). O material foi colhido no horto de plantas medicinais da Universidade Federal de Lavras (UFLA), na cidade de Lavras, região sul de Minas Gerais (Brasil), a $21^{\circ} 14^{\prime}$ S de longitude $45^{\circ} 00^{\prime}$ W GR, a $918 \mathrm{~m}$ de altitude. As plantas foram coletadas em novembro de 2008 , na parte da manhã, em dia com temperatura amena variando entre 22 e $25^{\circ} \mathrm{C}$ e sem precipitação pluviométrica. O material foi herborizado segundo técnicas convencionais e identificado pela Profa Dra Mariana Esteves Mansanares do

Rev. Bras. PI. Med., Botucatu, v.14, n.1, p.57-67, 2012. 
Departamento de Ecologia da Universidade Federal de Lavras, segundo literatura taxonômica especializada. Exemplares destas espécies encontram-se registradas no herbário da Universidade Federal de Lavras sob os seguintes números: 18407 (Mentha piperita L.), 18409 (Cymbopogon citrarus (D.C.) Stapf), 18408 (Origanum majorana L.) e 18406 (Ocimum basilicum L.).

\section{Extração de óleos essenciais}

O método empregado para extração dos óleos essenciais foi o de hidrodestilação utilizando o aparelho de Clevenger modificado (Glasstec, Glasslabor). Para tal, $75 \mathrm{~g}$ de folhas frescas de Mentha piperita L., Cymbopogon citrarus (D.C.) Stapf., Origanum majorana L., e Ocimum basilicum L. foram picadas e colocadas, juntamente com água em balão volumétrico com capacidade de $1.000 \mathrm{~mL}$. O balão foi acoplado ao aparelho de Clevenger modificado e a extração foi realizada pelo período de 2,5 horas, controlando-se a temperatura a, aproximadamente, $100^{\circ} \mathrm{C}$. Posteriormente, coletou-se o hidrolato, que foi centrifugado a $321,8 \times \mathrm{G}$, por 5 minutos. O óleo essencial foi retirado com auxílio de pipeta de Pasteur e estocado, à temperatura de refrigeração, em frascos de vidro envoltos por papel alumínio. (Guimarães et al., 2008).

\section{Identificação e quantificação dos constituintes químicos \\ Análises qualitativas dos óleos foram} realizadas por cromatografia em fase gasosa acoplada à espectrometria de massa (CG/EM), utilizando-se aparelho Shimadzu CG-17a, com detector seletivo de massa modelo QP $5050^{a}$, sob as seguintes condições operacionais: coluna capilar de sílica fundida (30 $\mathrm{mX}$ $0,25 \mathrm{~mm}$ ) com fase ligada DB-5MS (Folsom, CA, USA); temperatura da fonte de íons de $280^{\circ} \mathrm{C}$; programação da coluna com temperatura inicial de $50^{\circ} \mathrm{C}$, por 2 minutos com aumento de $4^{\circ} \mathrm{C}$ minuto ${ }^{-1}$ até $200^{\circ} \mathrm{C}$; depois, $10^{\circ} \mathrm{C}$ minuto ${ }^{-1}$ até $300^{\circ} \mathrm{C}$, finalizando com temperatura de $300^{\circ} \mathrm{C}$, por 10 minutos; gás carreador hélio $\left(1 \mathrm{~mL} \mathrm{~min}^{-1}\right)$; pressão inicial na coluna de $100,2 \mathrm{KPa}$; taxa split 1:83 e volume injetado de $1 \mu \mathrm{m}$ (1\% de solução em diclorometano). As condições para espectrômetro de massas (EM) foram energia de impacto de 70 eV, velocidade de decomposição 1000, intervalo de decomposição de 0,50 e fragmentos de $40 \mathrm{Da}$ e 550Da decompostos. Uma mistura linear de $\left(\mathrm{C}_{9} \mathrm{H}_{20} ; \mathrm{C}_{10} \mathrm{H}_{22} ; \mathrm{C}_{11} \mathrm{H}_{24} ; \ldots \mathrm{C}_{24} \mathrm{H}_{50} ; \mathrm{C}_{25} \mathrm{H}_{52} ; \mathrm{C}_{26} \mathrm{H}_{54}\right)$ foi injetada nas mesmas condições da amostra. Os espectros obtidos foram comparados com o banco de dados da biblioteca Wiley 229 e pelo índice de Kovats, calculando para cada constituinte de acordo com Adams (2007).

A quantificação dos constituintes dos óleos essenciais foi realizada por cromatografia em fase gasosa. Nesta análise, foi utilizado cromatógrafo gasoso Shimadzu CG - 17 A, com detector por ionização de chama (FID), nas condições cromatográficas de coluna capilar (DB5); programação da coluna com temperatura inicial de $50^{\circ} \mathrm{C}$, por 2 minutos, com aumento de $4^{\circ} \mathrm{C}$ minutos ${ }^{-1}$, até $200^{\circ} \mathrm{C}$; depois, $10^{\circ} \mathrm{C}$ minutos $^{-1}$ até $300^{\circ} \mathrm{C}$, finalizando com temperatura de $300^{\circ} \mathrm{C}$, por 10 minutos. Utilizou-se como gás carreador o nitrogênio $\left(2,2 \mathrm{~mL} \mathrm{~min}^{-1}\right)$ e, ainda, taxa de split de 1:10 e volume injetado de $1 \mu \mathrm{L}$ ( $1 \%$ de solução em diclorometano) e pressão na coluna de $115 \mathrm{KPa}$. A quantificação de cada constituinte foi obtida por meio de normalização de áreas (\%).

\section{Teste da atividade antimicrobiana Microrganismos}

Foram utilizadas cepas de Escherichia coli enteropatogênica CDC 0126, Salmonella entérica Enteritidis S64, Listeria monocytogenes ATCC 19117 e Enterobacter sakazakii ATCC 29004, provenientes da coleção de culturas da Fundação Oswaldo Cruz (Fiocruz).

\section{Padronização, estocagem e preparo do inóculo}

Para a padronização do número de células, a cepa foi inicialmente inoculada em frasco Erlenmeyer contendo $150 \mathrm{~mL}$ de meio de cultura caldo triptona de soja (TSB) (Himedia ${ }^{\circledR}$, Mumbai, Maharashtra, Índia), o qual foi incubado a $37^{\circ} \mathrm{C}$. Em seguida, a partir do TSB, a curva de crescimento foi determinada pela realização periódica de leituras da absorbância da cultura a $600 \mathrm{~nm}$ em espectrofotômetro (Bioespectro SP 22) e de diluições seriadas em água peptonada $0,1 \%(p / v)$ com posterior plaqueamento em superfície para determinação do Log UFC $\mathrm{mL}^{-1}$, utilizando-se como meio de cultura ágar triptona de soja (TSA) (Himedia $^{\circledR}$, Mumbai, Maharashtra, Índia).

Durante a realização do experimento, a cepa foi estocada sob refrigeração em meio de congelamento (por $100 \mathrm{~mL}$ de água destilada: 15 $\mathrm{mL}$ de glicerol, $0,5 \mathrm{~g}$ de peptona bacteriológica, $0,3 \mathrm{~g}$ de extrato de levedura e $0,5 \mathrm{~g}$ de $\mathrm{NaCl}, \mathrm{pH}$ $7,2 \pm 7,4)$. Para ativação e utilização da cepa, uma alíquota do meio de congelamento foi transferida para tubos de ensaio contendo TSB, sendo realizados dois repiques consecutivos com incubação a $37^{\circ} \mathrm{C}$, por 24 horas, neste meio de cultura. Em seguida, a cultura foi estriada em placas contendo TSA e incubada a $37^{\circ} \mathrm{C}$, por 24 horas. Das colônias formadas na superfície do TSA, foi retirada uma alçada e transferida para um frasco Elernmeyer contendo $150 \mathrm{~mL}$ de TSB, o qual foi incubado a $37^{\circ} \mathrm{C}$, até atingir o número de células necessárias para a utilização no experimento. 
Determinação da atividade antimicrobiana

A metodologia empregada foi a de difusão em ágar, de acordo com Mendonça (2004), com modificações. Como meio de cultura utilizou-se TSA. Para o preparo das cavidades de deposição dos óleos essenciais, uma camada inicial do meio de cultura foi depositada em placas de Petri, sobre as quais, após solidificação, foram dispostas pérolas de vidro. Uma sobrecamada do meio de cultura contendo o inócuo na concentração de, aproximadamente, $8 \mathrm{Log} \mathrm{UFCmL}^{-1}$, foi depositada sobre as pérolas de vidro dispostas na superfície da camada inicial. Após solidificação da sobrecamada, as pérolas de vidro foram removidas com o auxílio de pinças estéreis, dando origem às cavidades. Em seguida, $10 \mu \mathrm{l}$ das diferentes concentrações $(0 ; 0,39 ; 078 ; 1,56 ; 3,12 ; 6,25 ; 12,5$; $25 ; 50)$, expressas em $\%(\mathrm{v} / \mathrm{v})$ dos óleos essenciais de hortelã e capim-limão, manjerona, manjericão, diluídas em etanol a $95 \% \mathrm{v} / \mathrm{v}$, foram transferidas para as cavidades. A concentração $0 \%(\mathrm{v} / \mathrm{v})$, constituída apenas de etanol, foi utilizada como controle positivo do crescimento bacteriano. As placas foram incubadas, a $37^{\circ} \mathrm{C}$, por 24 horas e, após esse período, foram medidos os diâmetros dos halos de inibição formados com auxílio de um paquímetro. $\mathrm{O}$ experimento foi realizado em três repetições e os resultados foram expressos como a média dos três valores. A atividade antibacteriana foi caracterizada pelo comprometimento do crescimento bacteriano, observado pela formação de halo de inibição. A menor concentração que apresentou atividade antimicrobiana foi definida como concentração mínima inibitória (CMI).

\section{Análise dos dados}

Os dados de atividade bactericida dos óleos essenciais obtidos pelo método de difusão em placa foram padronizados em unidades arbitrarias por $\mathrm{mL}$ (UA mL-1) de acordo com Nguefack et al. (2004), para posteriormente, serem comparados entre si. Para a obtenção das UA $\mathrm{mL}^{-1}$ utilizou-se a fórmula:

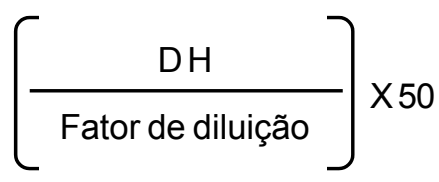

em que DH é o diâmetro inibitório para a menor concentração (em milímetros). De posse dos valores das $U A \mathrm{~mL}^{-1}$, procedeu-se a análise de variância, tendo os dados de UA dos óleos essenciais para cada bactéria sido transformados em raiz de $x+0,5$ e as medias comparadas pelo teste de Scott \& Knott (1974) usando o pacote "Laercio" (Silva, 2008), compilado pelo software $\mathrm{R}^{\circledR}$ (2009).

\section{RESULTADO E DISCUSSÃO}

A atividade antibacteriana dos óleos essenciais de Mentha piperita, Cymbopogon citratus, Ocimum basilicum e Origanum majorana contra as quatro espécies de bactérias testadas, Escherichia coli enteropatogênica, Salmonella entérica Enteritidis, Listeria monocytogenes e Enterobacter sakazakii está sumarizada na Tabela 1 . Os resultados revelam que os óleos essenciais demonstraram atividade antimicrobiana em variadas magnitudes, sendo a maioria das bactérias testadas sensível aos óleos.

Os principais constituintes do óleo essencial de $M$. piperita identificados e quantificados foram mentol $(32,33 \%)$, neoiso-mentol $(28,12 \%)$, mentona $(20,95 \%)$ acetato de metila $(6,65 \%)$ e iso-mentona $(4,82 \%)$ (Tabela 2$)$. Prévias investigações da composição são consistentes com os resultados encontrados, segundo os quais mentol e mentona são compostos majoritários. Iscan et al. (2002) elucidaram, por GC e GC/MS, a composição e relativa porcentagem de quatro amostras de óleos essenciais de $M$. piperita de fontes distintas. Os resultados demonstraram que as amostras continham mentol (28\%-42\%) e mentona ( $18 \%-28 \%)$ como os principais constituintes.

Quando comparada a atividade antibacteriana do óleo essencial de $M$. piperita frente às bactérias testada, observou-se que este apresentou maior atividade para $E$. coli, seguido de atividade moderada para Salmonella Enteritidis e Enterobacter sakazakii e baixa atividade para Listeria monocytogenes. Vários estudos têm avaliado a atividade antibacteriana de M. piperita (Cowan, 1999; Tassou et al., 2000, McKay \& Blumberg, 2006). Mentol tem sido relatado como sendo o composto responsável pela atividade antimicrobiana de M. piperita (Sivropoulou, 1995) e foi apresentado como ativo contra Clostridium sporogenes, Enterobacter aeraerogenes, Klebsiella pneumoniae, Proteus Vulgaris, Pseudomonas aeruginosa, Salmonella pullorum, Staphylococcus aureus e Streptococcus faecalis, inibindo estes microrganismos em concentrações de $10 \mu \mathrm{L}$ por placa, utilizando o método de plaqueamento em ágar (Iscan et al., 2002). Contudo, alguns estudos sugerem que mentol não parece ser o único agente responsável pela propriedade antimicrobiana de $M$. piperita. Yadegarinia et al. (2006) observaram alta atividade antimicrobiana de óleo essencial de $M$. piperita com concentrações baixas de mentol (3,6\%). Assim, podese deduzir que outros compostos químicos presentes no óleo também contribuem para alta atividade antimicrobiana do óleo essencial de M. piperita.

$\mathrm{Na}$ avaliação, o óleo essencial de $C$. citratus apresentou, na constituição, $38,43 \%$ de geranial, $31,12 \%$ de neral, $4,21 \%$ de linalol e $2,53 \%$ de mirceno (Tabela 2). Geranial e neral são estereoisômeros e a mistura constitui o citral. Assim, o citral é mistura

Rev. Bras. PI. Med., Botucatu, v.14, n.1, p.57-67, 2012. 
TABELA 1. Atividade bactericida $\left(\mathrm{UA} \mathrm{mL}^{-1}\right)$ dos diferentes óleos essenciais para Salmonella Enteritidis, Escherichia coli, Listeria monocytogenes e Enterobacter sakazakii.

\begin{tabular}{|c|c|c|c|c|c|c|}
\hline \multirow{3}{*}{ Bactéria } & \multicolumn{5}{|c|}{ Atividade do óleo essencial (UA mL)'1 } & \multirow{3}{*}{$\frac{\text { CV (\%) }}{10,48}$} \\
\hline & \multirow{2}{*}{$\frac{\text { Mentha piperita }}{1.706 \pm 282,21 \mathrm{~b} \mathrm{C}}$} & Cymbopogon citratus & \multirow{2}{*}{$\frac{\text { Ocimum basilicum }}{1.600 \pm 184,75 \text { b B }}$} & \multicolumn{2}{|c|}{ Origanum majorana } & \\
\hline & & $2.773 \pm 213,33$ a B & & $1.706 \pm 213,33$ & b B & \\
\hline 2 & $8.106 \pm 1.128 \quad$ aA & $9.386 \pm 853,33$ aA & $6.826 \pm 853,33$ aA & $5.973 \pm 426,67$ & aA & 9,79 \\
\hline 3 & $106,67 \pm 6,67$ b D & $2.773 \pm 213,33$ a B & $0,00 \pm 0,00$ & $0,00 \pm 0,00$ & c C & 10,85 \\
\hline 4 & $3.200 \pm 369,50$ b B & $2.773 \pm 213,33 \quad b \quad B$ & $8.106 \pm 426,67 \mathrm{aA}$ & $2.346 \pm 213,33$ & b B & 7,05 \\
\hline
\end{tabular}

Medias seguidas pela mesma letra minúscula na mesma linha ou maiúscula na mesma coluna, não diferem entre si, pelo teste de Scott \& Knott (1974) (pd"0,05). AU. $\mathrm{mL}^{-1}$ calculado usando a seguintes formula: media do diâmetro do halo de inibição $(\mathrm{mm}) \div$ fator diluição $\times 50$

isomérica de geranial [(2E)-3,7-dimetilocta-2,6-dienal; citral A ou isômero E] e neral [(2z)-3,7-dimetilocta2,6-dienal; citral B ou isômero Z] (El Fattah et al., 1992). Observa-se que os constituintes majoritários encontrados no óleo essencial de $C$. citratus utilizado neste estudo assemelha-se aos encontrados nos trabalhos consultados. Oliveira et al. (2010) encontraram, como constituintes do óleo essencial de C. citratus, geranial $(42,91 \%)$, neral $(30,90 \%)$, linalol $(1,51 \%)$ e mirceno $(1,36 \%)$.

O óleo essencial de Cymbopogon citratus apresentou maior atividade antimicrobiana frente a $E$. coli quando comparada as demais bactérias e atividade moderada frente a Enterobacter sakazakii, Salmonella enteritidis e Listeria monocytogenes.

Doran et al. (2009) demonstraram que o óleo essencial de $C$. citratus inibiu bactérias resistentes a antibióticos em concentrações muito baixas $(0,06 \%)$. Onawunmi (1984), ao avaliar a atividade de citral frente a bactérias e fungos, constatou que a mínima concentração inibitória obtida para $S$. aureus, E. coli, C. albicans e M. gypseum foi de $0,05 \%(\mathrm{v} / \mathrm{v})$, enquanto para $A$. fumigatus foi de $0,08 \%(\mathrm{v} / \mathrm{v})$. Segundo este autor, $0,01 \%$ de citral levou a ligeira inibição no crescimento de $E$. coli; já em concentrações mais altas $(0,03,0,05,0,08$ e $0,1 \%)$, citral demonstrou rápida diminuição na viabilidade da contagem final de células.

Entre os óleos essenciais avaliados, apenas C. citratus apresentou atividade frente à $L$. monocytogenes. Esses achados estão de acordo com os de Oussalah et al. (2007), que também observaram superioridade na atividade antibacteriana do óleo essencial de $C$. citratus frente a $L$. monocytogenes, tendo a CMl encontrada para o óleo essencial sido de 0,4\% (v/v). Lis-Balchin \& Deans (1997) observaram forte atividade antilisterial de $C$. citratus e encontraram correlação entre essa atividade e o alto conteúdo de geranial e neral. A moderada atividade registrada pelo método de difusão em Agar, neste estudo, pode ser atribuída à baixa afinidade entre componentes menos polares, como neral e geranial e o substrato polar (ágar), o que pode diminuir atividade do óleo essencial de $C$. citratus.

Apesar de as atividades antimicrobianas e antifúngicas do óleo essencial de $C$. citratus serem atribuídas ao citral (Guerra et al., 2000), é possível que a atividade dos principais componentes seja modulada por outras moléculas que estão presentes em menor quantidade. Onawunmi et al. (1984) demonstraram sinergismo entre os componentes do óleo, observando que mirceno não apresentou atividade antimicrobiana, mas, quando associado ao citral, potencializou o efeito. É possível que a atividade dos principais componentes seja modulada por moléculas menores (Hoet et al., 2006; Bakkali et al., 2008).

Óleo essencial de Ocimum basilicum apresentou maior atividade antimicrobiana para E. coli e Enterobacter sakazakii; moderada atividade frente a Salmonella Enteritidis e não apresentou atividade frente a Listeria monocytogenes. Segundo Sinha \& Gulati (1990), o óleo essencial de Ocimum basilicum de varias regiões foi efetivo contra Staphylococcus aureus, E. coli, Salmonella thyphi, Samonella paratyphi, Shigella boydii e Proteus vulgaris. Deans \& Ritcie (1987) ao avaliaram vários óleos essenciais (incluindo $O$. basilicum), quanto à propriedade antimicrobiana contra 25 gêneros de bactérias, utilizando a técnica de difusão em ágar, constataram que a maioria das bactérias testadas, incluindo Aeromonas hydrophila, B. subtilis, Brevibacterium linens, Brocothrix thermosphacta, Erwinia carotovora, E. coli, Lueconostoc cremoris, S. aureus, Streptococcus faecalis e Yersinia enterocolitica, apresentou ampla sensibilidade ao óleo essencial de O. basilicum.

O óleo essencial de $O$. basilicum apresentou na constituição $59,19 \%$ de linalol, $13,74 \%$ de 1,8 cineol, $6,91 \%$ de cinamato de metila, 3,94\% de áepi-cadinole e $2,44 \%$ de metil chavicol (Tabela 2). A atividade antibacteriana pode ser, em parte, devido, à presença do alto conteúdo de linalol (Sartoratto et al., 2004; Sokovic \& Griensven, 2006). 
A atividade antimicrobiana dos principais constituintes do óleo essencial de $O$. basilicum (Linalol, 1,8 cineol, metil chavicol, eugenol e metil cinamato) foi também estudada. Pattnaik et al. (1997) avaliaram as propriedades antibacterianas dos constituintes aromáticos de óleos essenciais. Os resultados dos testes demonstraram que linalol foi o mais efetivo composto, inibindo 17 das 18 cepas bacterianas. Álcoois são conhecidos por possuir atividade bactericida em vez de bacteriostática contra células vegetativas. $O$ álcool terciário linalol é ativo contra microrganismos testados, potencialmente atuando tanto como agente de desnaturação protéica ou como solvente desidratante (Suppakul et al., 2002).

Knobloch et al. (1989) avaliaram a atividade antimicrobiana dos componentes do óleo essencial de $O$. basilicum contra bactérias gram-negativas (e.g. Enterobacter aerogenas e $P$. Vulgaris), bactérias gram-positivas (e.g._. aureus e $B$. subtilis) e fungos (e.g. Aspergillus flavus, $A$. niger, $A$. ochraceus e $P$. expansum). Estes autores constataram que linalol, com alta solubilidade em água, teve significativa atividade antimicrobiana, quando comparado a outros compostos, como cinamaldeído, citral, geraniol, eugenol e mentol, ao passo que metil chavicol, com baixa solubilidade, apresentou menor atividade antimicrobiana.

A solubilidade em água dos constituintes dos óleos essenciais está diretamente relacionada à capacidade de penetrar na parede celular de fungos e bactérias. Contudo, a atividade antimicrobiana desses óleos deve-se à solubilidade na bicamada lipídica da membrana celular (Knobloch et al., 1989; Suppakul et al., 2002).

Óleo essencial de Origanum majorana também foi testado neste estudo e quando comparada sua atividade antibacteriana frente às bactérias, este apresentou maior atividade para E. coli, atividade moderada para Salmonella Enteritidis e Enterobacter sakazakii e não apresentou atividade antibacteriana para Listeria monocytogenes.

Para os constituintes do óleo essencial do O. majorana utilizado, as proporções e as substâncias identificadas foram $38,17 \%$ de terpinen-4-ol, $7,71 \%$ de $\gamma$-terpineno, $6,50 \%$ de $p$-cimeno e $3,84 \%$ de $\alpha$ terpineno (Tabela 2). Bussata et al. (2008), ao avaliarem a atividade antimicrobiana de óleo essencial de $O$. majorana frente a dez espécies de bactérias, observaram que todos os microrganismos testados foram suscetíveis à ação do óleo essencial, com valores de MIC a partir de 0,069 a $2.3 \mathrm{mg} \mathrm{mL}^{-1}$. Deans \& Svoboda (1990), em estudo sobre a atividade antimicrobiana do óleo de 0 . majorana sobre 25 bactérias, mostraram um poder inibitório sobre considerável número de bactérias.

A atividade antibacteriana apresentada pelo óleo pode ser devido à terpinen-4-ol e $\gamma$-terpineno. $\gamma$ Terpineno tem sido relatado por apresentar significativa atividade antimicrobiana (Carson \& Riley, 1995; Cosentino et al., 1999) e terpinen-4-ol tem sido citado por apresentar atividade bacteriostática contra alguns microrganismos (Barel et al., 1991). Contudo, os compostos que são encontrados em menor quantidade, como $p$-cimeno e $\alpha$-terpineol, podem também contribuir para atividade antimicrobiana dos óleos (Carson \& Riley, 1995; Pattnaik et al., 1997; Cosentino et al., 1999; Tabanca et al., 2001), sendo possível que estejam envolvidos em algum tipo de sinergismo com outros compostos ativos (Marino et al., 2001; Xianfei et al., 2007).

A maior atividade de óleo essencial frente a E. coli, Salmonella, Enteritidis e Enterobacter sakazakii e a menor atividade frente a Listeria monocytogenes diferem da maioria dos estudos que relatam que bactérias gram-positivas são mais sensíveis a óleos essenciais do que as gram-negativas (Davison, 1997). Entretanto, alguns estudos não confirmam estas observações. Burt (2004) afirma que bactérias gram-positivas têm sido menos ou igualmente sensíveis a bactérias gram-negativas. Doran et al. (2009), avaliando a atividade antimicrobiana de óleo essencial de $C$. citratus, demonstraram que este óleo inibiu bactéria resistente a antibiótico a baixas concentrações $(0,06 \%)$. Além disso, foi observado também que bactéria grampositiva foi menos susceptível do que gram-negativa. Ao estudarem a atividade antimicrobiana de 22 óleos essenciais frente a 10 cepas bacterianas, Kotzekidou et al. (2008) demonstraram que S. typhimurium (gramnegativa) foi a cepa mais sensível, sendo inibida por 17 dos compostos testados, enquanto $L$. monocytogenes (gram-positiva) foi a cepa mais resistente, sendo inibida por somente quatro dos compostos testados.

Os resultados do presente trabalho estão de acordo com estas observações, uma vez que Listeria monocytogenes apresentou maior resistência aos óleos testados quando comparada às bactérias gramnegativas. Randrianarivelo et al. (2009), ao avaliarem amostras de óleos essenciais de Cinnamosma fragrans originárias de duas regiões em Madagascar, Tsaramandrosa (B8) e Mariarano (B143), frente a bactérias gram-positivas e gram-negativas, observaram que cepas de Micrococcus foram mais resistentes aos óleos B134, sendo tão resistente quanto cepas de Salmonella ao óleo B8.

A permeabilidade da membrana bacteriana, a presença de proteínas porinas em bactérias gramnegativas e a distribuição intracelular dos constituintes dos óleos essenciais são elementos chaves que influenciam a difusão e a ação dos óleos essenciais dentro das células, sendo, então, a variação na atividade dos óleos essenciais esperada contra

Rev. Bras. Pl. Med., Botucatu, v.14, n.1, p.57-67, 2012. 
TABLE 2. Composição química dos óleos essenciais de Cymbopogon citratus, Mentha piperita, O. basilicum e O. majorana determinado por CG-MS.

\begin{tabular}{|c|c|c|c|c|}
\hline \multicolumn{5}{|c|}{ C. citratus } \\
\hline $\operatorname{TR}(\min )$ & Compostos & IRRexp & IRRlit & GC-MS(\%) \\
\hline 10,235 & metil-5-epten-2one & 986 & 981 & 0,43 \\
\hline 10,444 & mirceno & 990 & 990 & 2,53 \\
\hline 12,608 & linalol & 1100 & 1096 & 4,21 \\
\hline 20,897 & borneol & 1176 & 1165 & 0,24 \\
\hline 22,966 & neral & 1244 & 1240 & 31,12 \\
\hline 25,259 & geraniol & 1259 & 1249 & 1,33 \\
\hline 26,192 & geranial & 1278 & 1264 & 38,43 \\
\hline 27,073 & 2-undecanona & 1295 & 1293 & 2,37 \\
\hline \multicolumn{5}{|c|}{ Total identificado $80.66 \%$} \\
\hline \multicolumn{5}{|c|}{ M. piperita } \\
\hline $\mathrm{TR}(\mathrm{min})$ & Compostos & IRRexp & IRRlit & GC-MS(\%) \\
\hline 6,808 & artemisia triene & 932 & 929 & 0,32 \\
\hline 8,060 & sabineno & 971 & 975 & 0,12 \\
\hline 8,218 & $\beta$-pineno & 976 & 979 & 0,37 \\
\hline 8,616 & mirceno & 989 & 990 & 0,25 \\
\hline 9,996 & limoneno & 1028 & 1029 & 0,94 \\
\hline 10,109 & 1,8 -cineol & 1032 & 1031 & 0,07 \\
\hline 14,393 & isopulegol & 1149 & 1149 & 0,68 \\
\hline 14,712 & mentona & 1157 & 1152 & 20,95 \\
\hline 15,022 & iso-mentona & 1165 & 1162 & 4,82 \\
\hline 15,207 & mentol & 1170 & 1171 & 32,33 \\
\hline 15,688 & neoiso-menthol & 1183 & 1186 & 28,20 \\
\hline 17,695 & pulegona & 1238 & 1237 & 0,25 \\
\hline 18,269 & piperitona & 1238 & 1252 & 1,38 \\
\hline 18,890 & acetate de neo-isopulegila & 1271 & 1276 & 0,19 \\
\hline 18,942 & acetate neo-metila & 1273 & 1273 & 0,12 \\
\hline 19,586 & acetate de metila & 1290 & 1295 & 6,65 \\
\hline 20,108 & acetate de iso-metila & 1305 & 1305 & 0,16 \\
\hline 24,033 & (E)-cariofileno & 1419 & 1419 & 0,37 \\
\hline 26,064 & germacreno D & 1481 & 1481 & 0,15 \\
\hline \multicolumn{5}{|c|}{ Total identificado $98.32 \%$} \\
\hline \multicolumn{5}{|c|}{ O. basilicum } \\
\hline $\operatorname{TR}(\min )$ & Compostos & IRRexp & IRRlit & GC-MS(\%) \\
\hline 8,225 & $\beta$-pineno & 986 & 981 & 0,68 \\
\hline 10,117 & 1,8-cineol & 1031 & 1031 & 13,74 \\
\hline 12,608 & linalool & 1100 & 1096 & 59,19 \\
\hline 14,333 & cânfora & 1147 & 1146 & 0.86 \\
\hline 16,125 & a-terpineol & 1195 & 1188 & 1,52 \\
\hline 16,225 & metil chavicol & 1198 & 1196 & 2,44 \\
\hline 19,425 & acetate de isobornila & 1284 & 1285 & 0,96 \\
\hline 19,425 & trans-anetol & 1286 & 1284 & 0,90 \\
\hline 22,850 & cinamato de metila & 1383 & 1378 & 6,91 \\
\hline 23,383 & metil eugenol & 1399 & 1403 & 0,86 \\
\hline 27,100 & V-cadineno & 1513 & 1513 & 1,30 \\
\hline 31,083 & a-epi-cadinol & 1642 & 1640 & 3,94 \\
\hline \multicolumn{5}{|c|}{ Total identificado $93.3 \%$} \\
\hline
\end{tabular}


TABLE 2. Composição química dos óleos essenciais de Cymbopogon citratus, Mentha piperita, O. basilicum e $O$. majorana determinado por CG-MS.

...continuação

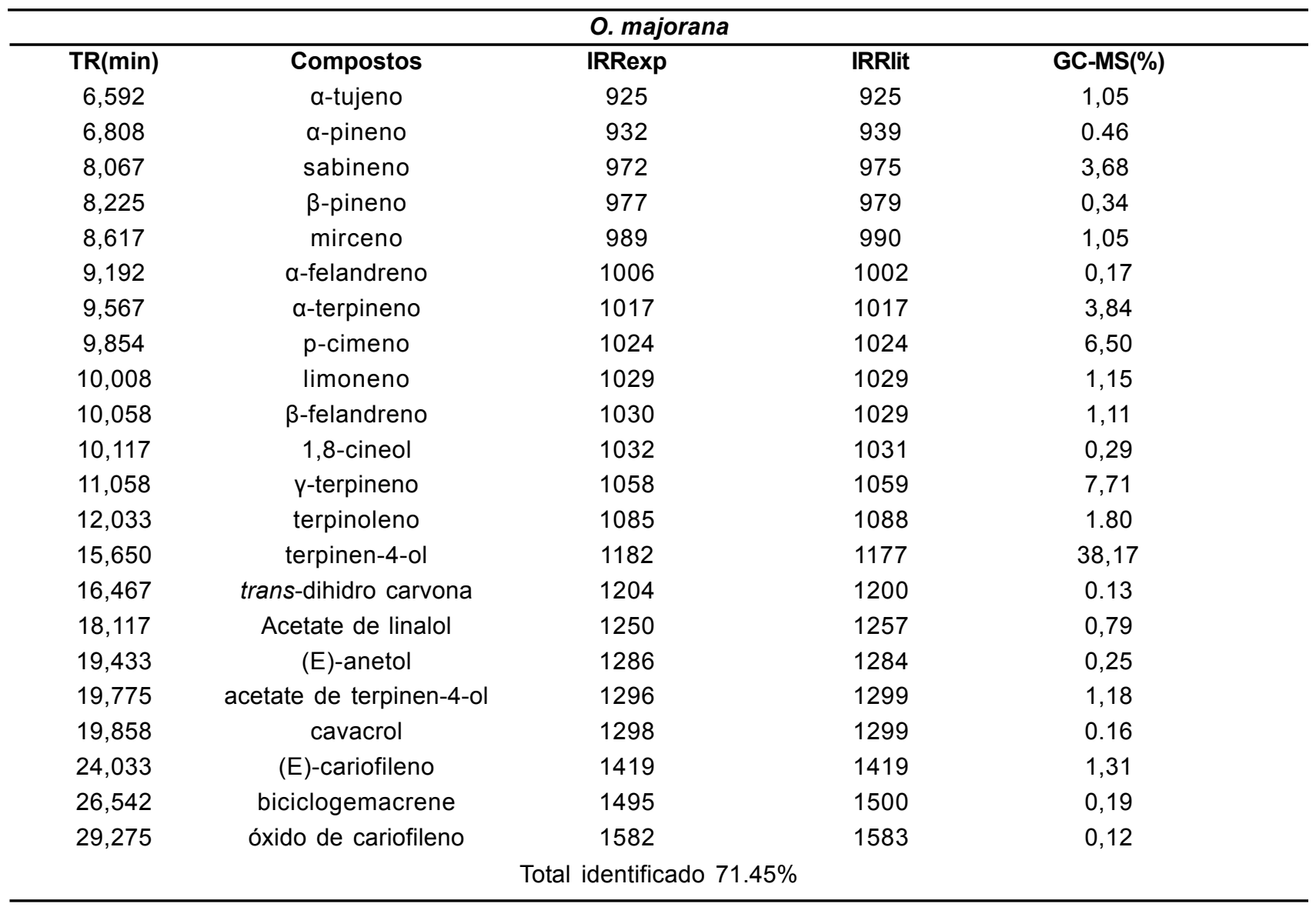

TR = tempo de retenção (minutos); IRRexp - índice experimental; IRRlit - índice literatura

diferentes grupos de bactérias (Lambert et al., 2001; Randrianarivelo et al., 2009).

Neste estudo foi também observado que, em geral, os óleos essenciais apresentam alta atividade antimicrobiana contra E. coli, concordando com outros estudos. Hammer et al. (1999), usando a técnica de difusão em Agar, concluíram que $E$. coli foi mais sensível do que cepa selecionada de $S$. typhimurium para a maioria dos óleos essenciais testados (Penalver et al., 2005).

A diferença encontrada entre a composição química dos óleos essenciais deste estudo e a composição química dos óleos essenciais de alguns trabalhos já realizados pode ser atribuída ao fato de que os óleos essenciais são um grupo heterogêneo de misturas de substâncias orgânicas nas quais os constituintes e as concentrações relativas não dependem somente da espécie da planta. Entre outros fatores que influenciam a composição química, os mais importantes são a origem da planta, a parte utilizada, o estágio de desenvolvimento, as condições climáticas e de crescimento, como temperatura, solo e fertilizantes e as condições de destilação e estocagem (Ozcan \& Erkmen, 2001; Oladimeji et al., 2001). Entretanto, a maioria componentes majoritários encontrados nos óleos essenciais estudados foi semelhante aos encontrados nos trabalhos consultados.

Essa diferença apresentada na constituição e concentração dos óleos essenciais influencia diretamente a atividade antimicrobiana. Assim, a diferença na atividade antimicrobiana existente entre os óleos essenciais de diferentes espécies de plantas, como observado entre os óleos essenciais de Mentha piperita, Cymbopogon citrarus, Origanum majorana e Ocimum basilicum), é relacionada à concentração e à natureza dos constituintes químicos (Chang et al., 1991).

Com relação aos óleos essenciais estudados, os constituintes majoritários encontrados foram monoterpenos. A ação antimicrobiana dos monoterpenos tem sido explicada pelo efeito tóxico na estrutura e função da membrana celular. Como resultado do caráter lipofílico, os monoterpenos irão, 
preferencialmente, se deslocar da fase aquosa em direção às estruturas da membrana (Sikkema et al., 1994), o que resulta em expansão da membrana, aumento da fluidez e permeabilidade da membrana, desordenando as proteínas embebidas na membrana, inibindo a respiração e alterando o processo de transporte de íons (Trombeta et al., 2009). Dessa forma, danos estruturais à membrana citoplasmática levam ao comprometimento das funções, como barreira seletiva e local de ação enzimática e geração de energia (Sikkema et al., 1994).

A capacidade apresentada pelos óleos essenciais em inibir o crescimento bacteriano permite que os mesmos sejam utilizados como antimicrobianos na indústria de alimentos.

\section{AGRADECIMENTO}

Os autores gostariam de agradecer CAPES, CNPq e FAPEMIG - pelo suporte financeiro.

\section{REFERÊNCIA}

Adams, R.P. Identification of essential oils components by gas chromatography / mass spectrometry. 4.ed. Carol Stream: Allured Publishing Corporation, 2007. 804p.

BAJPAI, V.K.; RAHMAN, A.; SUN C.K. Chemical composition and inhibitory parameters of essential oil and extracts of Nandina domestica Thunb. to control foodborne pathogenic and spoilage bacteria. International Journal of Food Microbiology, v.125, n.2, p.117-22, 2008. BAKKALI, F. et al. Biological effects of essential oil: a review. Food and Chemical Toxicology, v.46, n.2, p.44675, 2008.

BAREL, S.; SEGAL, R.; YASHPHE, J. The antimicrobial activity of the essential oil from Achillea fragrantissima. Journal of Ethnopharmacology, v.33, n.1/2, p.187-91, 1991.

BURT, S. Essential oils: their antibacterial properties and potential applications in foods - a review. International Journal of Food Microbiology, v.94, n.3, p.223-53, 2004. BUSATTA, C. et al. Application of Origanum majorana L. essential oil as an antimicrobial agent in sausage. Food Microbiology, v.25, n.1, p.207-11, 2008.

CARSON, C.F.; RILEY, T.V. Antimicrobial activity of the major components of the essential oil of Melaleuca alternifolia. Journal of Applied Bacteriology, v.78, n.3, p.264-9, 1995.

CHANG, W.C.; HSU, F.L. Inhibition of platelet activation and endothelial cell injury by polyphenolic compounds isolated from Lonicera japonica Thunb. Prostaglandins, Leukotrienes and Essential Fatty Acids, v.45, n.4, p.30712, 1991.

CIMANGA K. et al. Correlation between chemical composition and antibacterial activity of essential oils of some aromatic medicinal plants growing in the Democratic Republic of Congo. Journal of Ethnopharmacology, v.79 n.2, p.213-20, 2002
COSENTINO, S. et al. In-vitro antimicrobial activity and chemical composition of Sardinian Thymus essential oils. Letters in Applied Microbiology, v.29, n.2, p.130-5, 1999.

COWAN, M.M. Plant products and antimicrobial agents. Clinical Microbiology Reviews, v.12, n.4, p.564-82, 1999. DAVIDSON, P.M. Chemical preservatives and natural antimicrobial compounds. In: DOYLE, M.P.; BEUCHAT, L.R.; MONTVILLE, T.J. (Eds.). Food microbiology: fundamentals and frontiers. New York: AMS, 1997. p.52056.

DEANS, S.G.; RITCHIE, G. Antibacterial properties of plant essential oils. International Journal of Food Microbiology, v.5, p.165-80, 1987.

DEANS, S.G.; SVOBODA, K.P. The antimicrobial properties of marjoram (Origanim majorana L.) volatile oil. Flavour and Fragrance Journal, v.5, n.3, p.187-90, 1990.

DORAN, A.L. et al. Vapour-phase activities of essential oils against antibiotic sensitive and resistant bacteria including MRSA. Letters in Applied Microbiology, v.48, n.4, p.387-92, 2009.

DORMAN, H.J.D.; DEANS, S.G. Antimicrobial agents from plants: antibacterial activity of plant volatile oils. Journal of Applied Microbiology, v.88, n.2, p.308-16, 2000.

DRAUGHON, F.A. Use of botanicals as biopreservatives in foods. Food Technology, v.58, n.2, p.20-8, 2004.

EL FATTAH, M.A. et al. Effect of drying on the physicochemical properties and chemposition of lemongrass oil. Menofiya Journal Agriculture Research, v.17, n.3, p.1211-30, 1992.

FERREIRA, M.S.C.; FONTELES, M.C. Aspectos entnobotânicos e farmacológicos do Cymbopogon citratus Stapf (capim-limão). Revista Brasileira de Farmácia, v. 70, n.4, p.94-7, 1989

FILOCHE, S.K.; SOMA, K.; SISSONS, C.H. Antimicrobial effects of essential oils in combination with clorhexidine digluconate. Oral Microbiology and Immunology, v.20, n.4, p.221-5, 2005.

FRANTAMICO, P.M.; BHUNIA, A.K.J.; SMITH, L. Food-borne pathogens: microbiology and molecular biology. The Journal of Antimicrobial Chemotherapy, v.60, n.24, p.1180, 2007.

GUERRA, M.M.; BERNARDO, F.M.A. Relevância sanitária de Listéria monocytogenes nos produtos lácticos. Revista Portuguesa de Ciências Veterinária, v.94, n.530, p.142-8, 1999.

GUIMARÃES, L.G.L. et al. Influência da luz e da temperatura sobre a oxidação do óleo essencial de capim-limão (Cymbopogon citratus (DC.) Stapf). Química Nova, São Paulo, v.3, n.6, p.1476-80, 2008.

HAMMER, K.A.; CARSON, C.F.; RILEY, T.V. Antimicrobial activity of essential oils and other plant extracts. Journal of Applied Microbiology, v.86, n.6, p.985-90, 1999.

HOET, S. et al. Antitrypanosomal compounds from leaf essential oil of Strychnos spinosa. Planta Medica, v.72, n.5, p.480-2, 2006.

ISCAN, G. et al. Antimicrobial screening of Mentha piperita essential oils. Journal of Agricultural and Food Chemistry, v.50, n.14, p.3943-6, 2002.

$\mathrm{KNOBLOCH}$, K. et al. Antibacterial and antifungal properties of essential oil components. Journal of Essential Oil Research, v.1, n.1, p.119-28, 1989.

KOUTSOUDAKI, C.; KRSEK, M.; RODGER, A. Chemical

Rev. Bras. PI. Med., Botucatu, v.14, n.1, p.57-67, 2012. 
composition and antibacterial activity of the essential oil and the gum of Pistacia lentiscus Var. chia. Journal of Agricultural and Food Chemistry, v.53, n.20, p.7681-5, 2005.

LAMBERT, R.J.W. A study of the minimum inhibitory concentration and mode of action of oregano essential oil, thymol and carvacrol. Journal of Applied Microbiology, v.91, n.3, p.453-62, 2001.

LEE, S.J. et al. Identification of volatile components in basil (Ocimum basilicum L.) and thyme leaves (Thymus vulgaris L.) and their antioxidant properties. Food Chemistry, v.91, n.1, p.131-7, 2005.

LIS-BALCHIN, M.; DEANS, S.G. Bioactivity of selected plant essential oils against Listeria monocytogenes. Journal of Applied Bacteriology, v.82, n.6, p.759-62, 1997.

MARINO, M.; BERSANI, C.; COMI, G. Impedance measurements to study the antimicrobial activity of essential oils from lamiaceace and compositae. International Journal of Food Microbiology, v.67, n.3, p.187-95, 2001.

MARTINS, E.R. et al. Plantas medicinais. Viçosa, MG: UFV, 2003. 220p.

MCKAY, D.; BLUMBERG, J.B. A review of the bioactivity and potential health benefits of chamomile tea (Matricaria recutita L.). Phytotherapy Research, v.20, n.7, p.519-30, 2006.

MENDONÇA, A.T. Efeito dos óleos essenciais de condimentos sobre o crescimento de Staphylococcus aureus em ricota cremosa. 2004. 72p. Tese (Doutorado Área de concentração em Ciência dos Alimentos) Departamento de Ciências dos Alimentos Universidade Federal de Lavras, Lavras.

NEDOROSTOVA, L. et al. Antimicrobial properties of selected essential oils in vapour phase against foodborne bacteria. Food Control, v.20, n.2, p.57-60, 2009.

NGUEFACK, J.; BUDDE, B.B.; JAKOBSEN, M. Five essential oils from aromatic plants of Cameroon: their antibacterial activity and ability to permeabilize the cytoplasmic membrane of Listeria innocua examined by flow cytometry. Letters in Applied Microbiology, v.39, P.395-400, 2004.

OLADIMEJI, F.A. et al. Effect of autoxidation on the composition and antimicrobial activity of essential oil of Lippia multiflora. Pharmaceutical and Pharmacological Letters, v.11, n.2, p.64-7, 2001.

OLIVEIRA, M.M.M. et al. Disinfectant action of Cymbopogon sp. essential oils in different phases of biofilm formation by Listeria monocytogenes on stainless steel surface. Food Control, v.21, n.4, p.54953, 2010.

OLIVEIRA, T. L. C. et al. Antimicrobial activity of Satureja montana $\mathrm{L}$. essential oil against Clostridium perfringens type A inoculated in mortadella-type sausages formulated with different levels of sodium nitrite. International Journal of Food Microbiology, v.144, n.3, 2011.

ONAWUNMI, G.O. Evaluation of the antimicrobial activity of citral. Letters in Applied Microbiology, v.9, n.3, p.1058, 1984.

OUSSALAH, M. et al. Inhibitory effects of selected plant essential oils on the growth of four pathogenic bacteria: E. coli 0157:H7, Salmonella Typhimurium, Staphylococcus aureus and Listeria monocytogenes. Food Control, v.18, n.5, p.414-20, 2007.

OZCAN, M.; ERKMEN, O. Antimicrobial activity of the essential oils of Turkish plant spices. European Food Research and Technology, v.212, n.6, p.658-60, 2001. PATTNAIK, S. et al. Antibacterial and antifungal activity of aromatic constituents of essential oils. Microbios, v.89, n.358, p.39-46, 1997.

PENALVER, P. et al. Antimicrobila activity of five essential oils against origin strains o the Enterobacteriaceae family. Acta pathologica, microbiologica, et immunologica Scandinavica, v.113, n.1, p.1-6, 2005.

R DEVELOPMENT CORE TEAM. R: a language and environment for statistical computing. Viena: R Foundation for Statistical Computing, 2009. Disponível em: <http://www. R - project. org>. Acesso em: 20 out. 2009.

RANDRIANARIVELO, R. et al. Composition and antimicrobial activity of essential oils of Cinnamosma fragrans. Food Chemistry, v.114, n.2, p.680-4, 2009.

RICKE, S.C. et al. Alternatives to antibiotics: chemical and physical antimicrobial interventions and food-borne pathogen response. Poultry Science, v.84, n.4, p.66775, 2005.

SARTORATTO, A. et al. Composition and antimicrobial activity of essential oils from aromatic plants used in Brazil. Brazilian Journal of Microbiology, v.35, n.4, p.27580, 2004.

SCOTT, A.J.; KNOTT, M. A cluster analysis method for grouping means in the analysis of variance. Biometrics, v.30, n.3, p.507-12, 1974.

SELLAMI, I.H. et al. Effect of growth stage on the content and composition of the essential oil and phenolic fraction of sweet marjoram (Origanum majorana L.), Industrial Crops and Products, v.30, n.3, p.395-402, 2009.

SIKKEMA, J.; DE BONT, J.A.M.; POOLMAN, B. Interactions of cyclic hydrocarbons with biological membranes. The Journal of Biological Chemistry, v.269, p.8022-8, 1994. SILVA, L.J. Laercio: duncan test, tukey test and scottknott test. R package version 1.0-0, 008. 2008. 5p.

SINGH, R.; SHUSHNI, M.A.M.; BELKHEIR, A. Antibacterial and antioxidant activity of Mentha piperita L., Arabian Journal of Chemistry, v.4, n.1, p.1-20, 2011

SINHÁ, G.K.; GULATI, B.C. Antibacterial and antifungal study of some essential oils and some of their constituents. Indian Perfumer, v.34, n.2, p.126-9, 1990. SIVROPOULOU, A.; KOKKINI, S.; LANARAS, T. Antimicrobial activity of mint essential oil. Journal of Agricultural and Food Chemistry, v.43, n.9, p.2384-8, 1995.

SOKOVIC, M.; GRIENSVEN, L.J.L.D. van. Antimicrobial activity of essential oils and their components against the three major pathogens of the cultivated button mushroom, Agaricus bisporus. European Journal of Plant Pathology, v.116, n.3, p.211-24, 2006.

SOLOMAKOSA, N.; GOVARISA, A.; KOIDISB, P.; BOTSOGLOU, N. The antimicrobial effect of thyme essencial oil, nisin, and their combination against Listeria monocytogenes in minced beef during refrigerated storage. Food Microbiology, v.25, n.1, p.1207, 2008.

SUPPAKUL, P. et al. Preliminary stud of antimicrobial films the containing principal constituents of basil. In: IAPRI WORLD CONFERENCE ON PACKAGING, 13., 2002, East Lansing. Proceeding... East Lansing: CRC, 2002. 
TABANCA, N. et al. Composition and antimicrobial activity of the essential oils of Micromeria cristata subsp. phyrgia and the enantiomeric distribution of borneol. Journal of Agricultural and Food Chemistry, v.49, n.9, p.4300-3, 2001. TASSOU, C.C.; KOUTSOUMANIS, K.; NYCHAS, G.J.E. Inhibition of Salmonella enteritidis and Staphylococcus aureus in nutriente broth by mint essential oil. Food Research International, v.33, n.3/4, p.273-80, 2000.

TELCI, I.; BAYRAM, E.; YÝLMAZ, G.; AVCÝ, B. Variability in essential oil composition of Turkish basils (Ocimum basilicum L.) Biochemical Systematics and Ecology, v.34, n.6, p.489-97, 2006.

TROMBETTA, D. et al. Mechanisms of antibacterial action of three monoterpenes. Antimicrobial Agents and Chemotherapy, v.49, n.6, p.2474-8, 2005.

XIANFEI, X. et al. Chemical composition and antimicrobial activity of essential oils of Chaenomeles speciosa from China. Food Chemistry, v.100, n.4, p.1312-5, 2007.

YADEGARINIA, D. et al. Biochemical activities of Iranian Mentha piperita L. and Myrtus communis L. essential oils. Phytochemistry, v.67, n.12, p.124955, 2006. 